\title{
The mitogenome of Onchocerca volvulus from the Brazilian Amazonia focus
}

\author{
James L Crainey ${ }^{1}$, Túllio RR da Silva ${ }^{1}$, Fernando Encinas²+, \\ Michel A Marín², Ana Carolina P Vicente², Sérgio LB Luz ${ }^{1}$ \\ ${ }^{1}$ Fundação Oswaldo Cruz, Instituto Leônidas e Maria Deane, Laboratório de Ecologia de Doenças Transmissíveis na Amazônia, Manaus, AM,

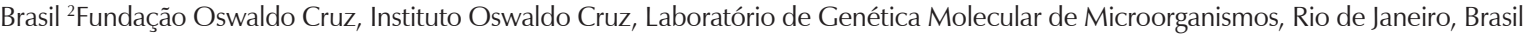

We report here the first complete mitochondria genome of Onchocerca volvulus from a focus outside of Africa. An O. volvulus mitogenome from the Brazilian Amazonia focus was obtained using a combination of highthroughput and Sanger sequencing technologies. Comparisons made between this mitochondrial genome and publicly available mitochondrial sequences identified 46 variant nucleotide positions and suggested that our Brazilian mitogenome is more closely related to Cameroon-origin mitochondria than West African-origin mitochondria. As well as providing insights into the origins of Latin American onchocerciasis, the Brazilian Amazonia focus mitogenome may also have value as an epidemiological resource.

Key words: Onchocerca volvulus mitochondria - onchocerciasis - Brazilian Amazonia focus - mitogenome

Onchocerca volvulus is the sole cause of onchocerciasis in humans, and humans are the parasites' only known reservoir (Crainey et al. 2016, unpublished observations). The Amazonia onchocerciasis focus is the last of the Latin American foci where O. volvulus transmission is still considered to be on-going and as such poses the principal source of risk for new infections (Luz et al. 2014, Crainey et al. 2016, unpublished observations). As mitochondria usually occur in very high copy number within animal cells, they have become a popular target for both parasite and vector molecular diagnostics, taxonomy, and population studies (Post et al. 2009, Conceição et al. 2013, Crainey et al. 2014). The availability of a complete O. volvulus mitogenome from South America provides a unique opportunity to characterise single nucleotide variant (SNV) loci as well as glean new insights into the parasites' epidemiological and evolutionary history.

The $O$. volvulus DNA used in this study derived from a skin biopsy taken from a resident of the Brazilian Amazonia focus; parasite DNA was isolated from $O$. volvulus following procedures described previously (MoralesHojas \& Post 2000, Ta Tang et al. 2010). Whole-genome sequencing was performed on an Illumina HiSeq 2500 system (Oswaldo Cruz Foundation, high-throughput sequencing platform) using $2 \times 100 \mathrm{bp}$ paired-end reads generated with Nextera Truseq libraries. Reads corresponding to human-host DNA were filtered out by mapping them against a human reference genome (acces-

doi: 10.1590/0074-02760150350

Financial support: FAPEAM, BSM/CAPES, PNPD/CAPES

+ Corresponding author: fencinas@ioc.fiocruz.br

Received 15 September 2015

Accepted 19 November 2015 sion GCA_000001405.19). An indexed database of $O$. volvulus genomic sequences (RefSeq; National Center for Biotechnology Information) was constructed to align reads that did not map to the human reference sequences. An $O$. volvulus mitochondria complete genome (accession AF015193) originating from West Africa was used as reference to extract mitochondrial reads corresponding to the Brazilian $O$. volvulus mitogenome (mtOvBz). The assembly of the mtOvBz genome was achieved with the A5-miseq pipeline (arxiv.org/abs/1401.5130). Mapping and short read post-processing were performed using Bowtie2 software (bowtie-bio.sourceforge.net/ bowtie2) and Samtools utilities (htslib.org/), respectively. Protein-coding genes, rRNAs, and tRNAs prediction were performed using the MITOS server (mitos.bioinf. uni-leipzig.de/index.py) and Arwen software (mbioserv2.mbioekol.lu.se/ARWEN/) followed by manual validation comparing homolog regions with the mitochondria reference in Artemis (Rutherford et al. 2000). A mitogenome map was generated using the BRIG software package (brig.sourceforge.net). Polymerase chain reaction and direct Sanger sequencing was used to confirm mtOvBz alleles at 45 of the 46 identified SNV loci (Table). Primer3 software (bioinfo.ut.ee/primer3-0.4.0) was used to design 14 primers sets for this purpose.

A total of 71,936 reads were used in the assembly of a single mitochondria genome contig of $13,769 \mathrm{bp}$, with $\sim 500 \mathrm{x}$ sequencing coverage. The $\mathrm{mtOvBz}$ genome is the first from Latin America, the first from outside of Africa, and only the second $O$. volvulus mitochondria genome to be completed. This genome is available from GenBank under accession KT599912. Its total GC content is $26.7 \%$, with base composition of $19.3 \% \mathrm{~A}, 54 \% \mathrm{~T}$, $19.9 \% \mathrm{G}$, and $6.8 \% \mathrm{C}$. It has 36 genes (12 protein-coding, 22 tRNA and 2 rRNA genes) and a 294 bp noncoding AT-rich region. The $\mathrm{mtOvBz}$ is identical in gene content and structure to the West African mitogenome and differs from it at just 37 nucleotide positions (Figure). 


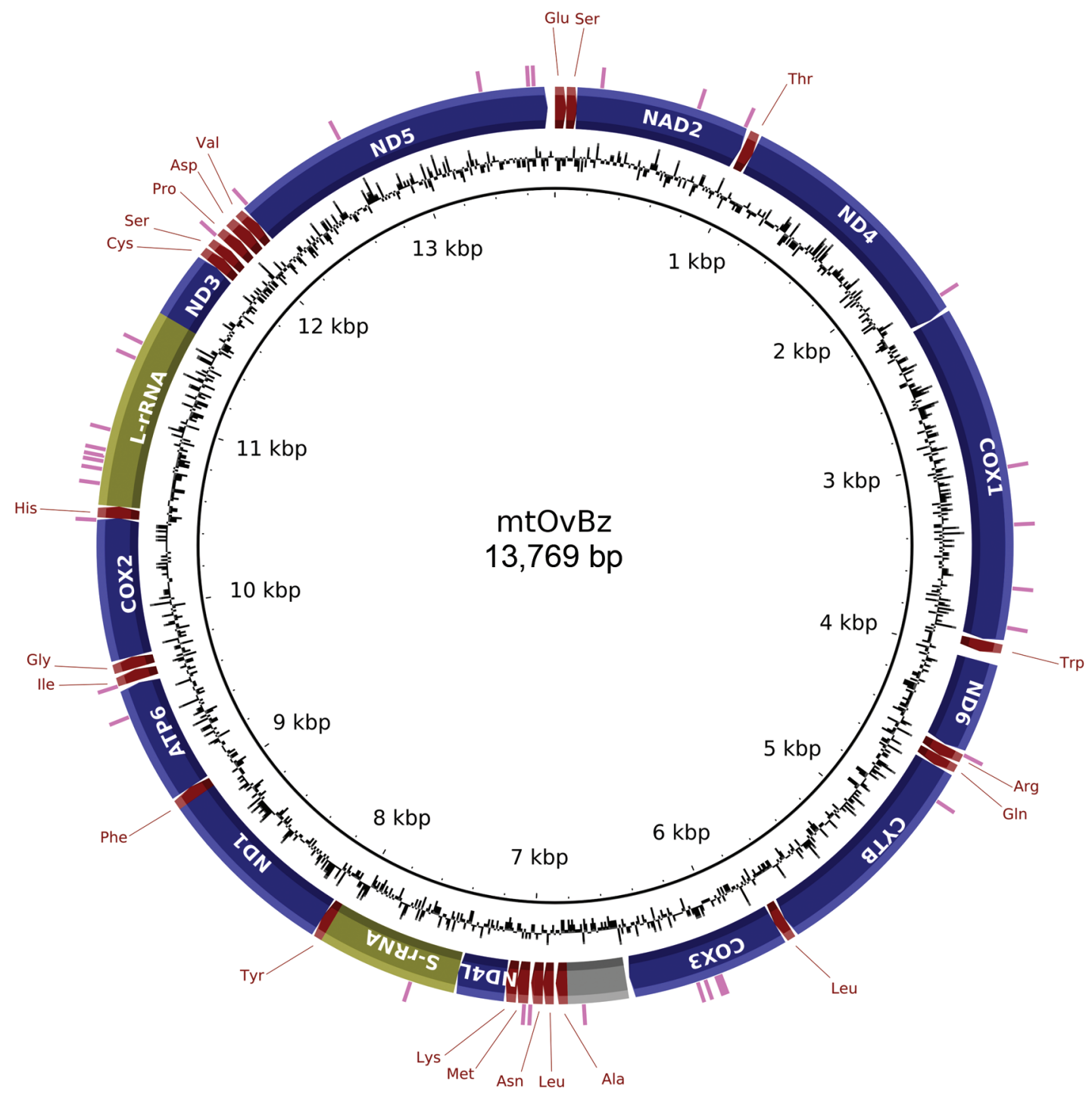

Complete mitogenome of Onchocerca volvulus from the Brazilian Amazonia focus (mtOvBz). The 13,769 bp mitogenome structure and content is visualised in a circular map. Protein-coding genes, tRNA, and rRNA genes are depicted in blue, maroon, and olive, respectively. AT-rich region and single nucleotide variations are shown in grey and magenta.

Interestingly, L-rRNA is the gene with most SNV loci; the ND5 gene has the next most, followed by the COX1 and $\mathrm{COX} 3$ genes. Comparing the Brazilian and West African origin mitogenomes with a mitochondria draft genome from Cameroon (accession HG738213.2) and other publicly available sequences we have been able to identify a further nine variant loci, bringing the total number of known/putative variant loci to 46. Considering the available sequence from Cameroon, our analysis showed that this mitogenome is missing $~ 185$ nucleotides corresponding to the tail end of the ND4 gene, immediately before the COX1 gene (nucleotide positions 2,076-2,259 within the AF015193 reference genome).
Comparative mitogenomic analysis also revealed that although all $O$. volvulus (West African, Cameroon, and Brazilian Amazonia focus) mitochondria share $\sim 99 \%$ of sequence identity, the mtOvBz genome is more closely related to the Cameroon-origin mitogenome than it is to the West-Africa mitogenome. Comparing alleles at the $43 \mathrm{SNV}$ loci for which data is available for all three mitogenomes, the mtOvBz genome and the Cameroonorigin mitogenome can be seen to differ at just 20 loci whereas the $\mathrm{mtOvBz}$ and West African-origin mitogenomes at 36 loci. Consistent with the notion that Latin American O. volvulus parasites diverged from African parasites after the species radiated in Africa, West Af- 
TABLE

Polymerase chain reaction primers used to verify Brazilian Amazonia focus alleles at 45 variable loci

\begin{tabular}{|c|c|c|c|}
\hline Forward primer & Reverse primer & $\begin{array}{l}\text { Amplicon size } \\
\text { (bp) }\end{array}$ & $\begin{array}{l}\text { Coordinates of verified } \\
\text { single nucleotide variations }\end{array}$ \\
\hline TGTTTCGTGTGGGAGCTTTT & TGCAACTTCCAACCATCAAA & 812 & $226,13411,13639,13665,13741$ \\
\hline TGGGCTCTGCTGAATCTTTT & AACAAACAACCAAACCAGGAA & 582 & $698,937,1052$ \\
\hline TGTTTTGTTTGATGTTTTGTTTGA & CCACCTAAACCAGCCCAATA & 410 & 2182 \\
\hline GGGTGGTCCTGGTAGGAGTT & ATCCAAACTAGCAGCCCTCA & 733 & $3066,3306,3343$ \\
\hline TTGCTGGTTTACAGGGTAT & CTTTTCAACGGATCCCAAT & 517 & 3648,3837 \\
\hline TTGTTGTAGATTTTGATTTTTCTTTG & AАAАСТСССССАААТССАТС & 751 & 4481,4735 \\
\hline TGTGGATTAAGGATGTTATTTTAGAGG & AGTTGAACAACTTAACACGAAAAA & 507 & $6079,6094,6101,6155,6193$ \\
\hline TTTCTTTGTTGTGGAGGGATA & AAAAACAAAAATTCAATACCCAAC & 709 & $6748,6749,7002,7032$ \\
\hline TTTTAAGTTTGATTTTGGTTTAGGTTG & ATGTGCCAACAAAATTCACC & 401 & 7586,7596 \\
\hline GTGAGCTGGTAAGGGGGTTT & AACAACTCCACCGGAACAAC & 856 & 8860,9488 \\
\hline TTTTGACTTTGGTTTGTATGTTTTTA & ATAAATCCCGCCACTAACCA & 311 & 9643,9644 \\
\hline TCGGTGTGTTTTGCCTGTAG & CACGCTAAGGCTGCCATTTA & 789 & $\begin{array}{c}10303,10449,10624,10688,10693 \\
10731,10756,10787,10885\end{array}$ \\
\hline TTTTCTTGGGGATGGATTTT & AATCCAAACGCCCCTAACAT & 837 & $11246,11324,11947$ \\
\hline GTTGTCTGCAAATAGGATTTGAT & TGCAAACCCCTACCAATAGC & 913 & 12157,12697 \\
\hline
\end{tabular}

coordinates are all in reference to the first published Onchocerca volvulus mitogenome (AF015193). Nucleotide insertions occur immediately after their coordinates.

rican-origin and Cameroon-origin mitogenomes vary at 30 of these 43 nucleotide positions and thus appear more diverged (from one another) than the mtOvBz and Cameroon-origin mitogenomes are (Morales-Hojas et al. 2006, 2007, Morales-Hojas 2009).

The low-levels of genetic variation detected in this study are consistent with previous studies of the parasite's evolutionary history that have concluded that onchocerciasis is a relatively new form of human parasitism (Keddie et al. 1998, 1999, Morales-Hojas et al. 2006, 2007, Morales-Hojas 2009). The SNVs identified in the present study may represent useful new markers for $O$. volvulus population studies and could therefore provide an important resource to obtain a clearer picture of $O$. volvulus epidemiology to aid with onchocerciasis elimination from the Amazonia focus.

\section{REFERENCES}

Conceição PA, Crainey JL, Almeida TP, Shelley AJ, Luz SLB 2013. New molecular identifiers for Simulium limbatum and Simulium incrustatum s.l. and the detection of genetic substructure with potential implications for onchocerciasis epidemiology in the Amazonia focus of Brazil. Acta Trop 127: 118-125.

Crainey JL, Mattos-Glória A, Hamada N, Luz SLB 2014. New tools and insights to assist with the molecular identification of Simulium guianense s.l., main Onchocerca volvulus vector within the highland areas of the Amazonia onchocerciasis focus. Acta Trop 131: 47-55.

Crainey JL, Medeiros JF, Pessoa FAC, Luz SLB 2016. Onchocerciasis. In CBG Marcondes (ed.), Arthropod borne disease, Springer, Heidelberg, ISBN 978-3-319-13884-8, in press.

Keddie EM, Higazi T, Boakye D, Merriweather A, Wooten MC, Unnasch TR 1999. Onchocerca volvulus: limited heterogeneity in the nuclear and mitochondrial genomes. Exp Parasitol 93: 198-206.
Keddie EM, Higazi T, Unnasch TR 1998. The mitochondrial genome of Onchocerca volvulus: sequence, structure and phylogenetic analysis. Mol Biochem Parasitol 95: 111-127.

Luz SLB, Crainey JL, Shelley AJ, Rubio JM 2014. Outstanding insecurities concerning the use of an Ov16-based ELISA in the Amazonia onchocerciasis focus. Mem Inst Oswaldo Cruz 109: 506-510.

Morales-Hojas R 2009. Molecular systematics of filarial parasites, with an emphasis on groups of medical and veterinary importance and its relevance for epidemiology. Infect Genet Evol 9: 748-759.

Morales-Hojas R, Cheke RA, Post RJ 2006. Molecular systematics of five Onchocerca species (Nematoda: Filarioidea) including the human parasite, $O$. volvulus, suggest sympatric speciation. $J$ Helminthol 80: 281-290.

Morales-Hojas R, Cheke RA, Post RJ 2007. A preliminary analysis of the population genetics and molecular phylogenetics of Onchocerca volvulus (Nematoda: Filarioidea) using nuclear ribosomal second internal transcribed spacer sequences. Mem Inst Oswaldo Cruz 102: 879-882.

Morales-Hojas RM, Post RJ 2000. Regional genetic variation in the major sperm protein genes of Onchocerca volvulus and Mansonella ozzardi (Nematoda: Filarioidea). Int J Parasitol 30: 1459-1465.

Post RJ, Crainey JL, Bivand A, Renz A 2009. Laser-assisted microdissection for the study of the ecology of parasites in their hosts. Mol Ecol Resou 9: 480-486.

Rutherford K, Parkhill J, Crook J, Horsnell T, Rice P, Rajandream MA, Barrell B 2000. Artemis: sequence visualization and annotation. Bioinformatics 16: 944-945.

Ta Tang T-H, López-Vélez R, Lanza M, Shelley AJ, Rubio JM, Luz SLB 2010. Nested PCR to detect and distinguish the sympatric filarial species Onchocerca volvulus, Mansonella ozzardi, and Mansonella perstans in the Amazon Region. Mem Inst Oswaldo Cruz 105: 823-828. 